\title{
Inhibition of saccadic eye movements to locations in spatial working memory
}

\author{
Artem V. Belopolsky AND Jan TheEUWeS \\ Vrije Universiteit, Amsterdam, The Netherlands
}

\begin{abstract}
Inhibition of return (IOR) refers to a bias against overt and covert attentional orienting toward previously attended locations. According to the reorienting hypothesis, IOR is generated when attention is withdrawn from the attended location and is prevented from "returning" to it. The present study investigated whether maintenance of attention at the cued location could affect the inhibition of oculomotor orienting to it. To preclude disengagement of attention, we asked participants to maintain the cued location in working memory. Maintenance of visuospatial information in memory has been shown to be accomplished through a sustained shift of spatial attention to a memorized location. Our results show that oculomotor IOR occurs at a particular location even when that location is kept in working memory (Experiment 1). Furthermore, we demonstrate that the mere act of maintenance of a location in working memory produces oculomotor inhibition similar to IOR (Experiments 2 and 3). We conclude that the oculomotor system is used for coding and maintaining locations in spatial working memory. In addition, we demonstrate that endogenous attention associated with maintenance of a location in working memory can be dissociated from the attention needed for execution of a saccadic eye movement.
\end{abstract}

A long line of research has demonstrated that allocation of attention in visual space results in enhancement of processing at the attended location, as measured on both behavioral and neural levels (Mangun et al., 2001; Posner, 1980). Attention is often viewed as a single spotlight that either can be oriented to a location strategically or endogenously or can be summoned automatically, exogenously, to a location of a salient external stimulus in the environment (Posner, 1980). The time courses of the two forms of attentional orienting are very different. Whereas endogenous attention produces a long-lasting facilitation of processing, a biphasic pattern of facilitation followed by inhibition of processing at the attended location is often considered the hallmark of exogenous orienting (Posner, 1980).

This inhibitory effect, specific to exogenous attention, had been termed inhibition of return (IOR), implying both a phenomenon and a mechanism by which attention is biased from returning to a previously attended location (Posner, Rafal, Choate, \& Vaughan, 1985). Such a mechanism is proposed to have a strong adaptive value, ensuring that sampling of novel locations is favored over returning to locations that have already been inspected (Klein, 2000). One of the critical assumptions of this reorienting hypothesis of IOR is that attention first has to be disengaged or withdrawn from the attended location before it is inhibited from returning to it in the future.

Generation of IOR has been strongly linked to the mechanisms and brain structures involved in eye movement control (Dorris, Klein, Everling, \& Munoz, 2002;
Ro, Farne, \& Chang, 2003; Sapir, Soroker, Berger, \& Henik, 1999). Many researchers have shown that inhibition increases saccadic latencies to previously covertly or overtly attended locations (Briand, Larrison, \& Sereno, 2000; Klein \& MacInnes, 1999) and biases saccades away from these locations (Posner et al., 1985). In an influential study by Rafal and colleagues (Rafal, Calabresi, Brennan, \& Sciolto, 1989), it was demonstrated that the eye movement system is directly involved in the generation of IOR. Although IOR is typically not found when attention is allocated endogenously, they showed that endogenous programming of a saccade resulted in inhibition of the processing of targets occurring at that location even when the programmed saccade was canceled rather than executed. Further evidence for the motor component of IOR was provided in a study by Abrams and Dobkin (1994; see also Posner et al., 1985). In order to isolate the motor locus of inhibition, an arrow at the fovea was used as the imperative signal indicating where an eye movement had to be made. This allowed participants to voluntarily program an eye movement, instead of being driven by a peripheral target. The results showed that saccades were initiated more slowly to the cued location, relative to other locations. This finding clearly suggests that covert attentional orienting can result in inhibition of the mechanisms involved in saccade production.

In the present study, we used a method similar to the one used by Abrams and Dobkin (1994) to examine the relationship between the oculomotor component of IOR and attentional disengagement. Specifically, we were interested

A.V. Belopolsky, a.belopolsky@psy.vu.nl 
in whether disengagement of attention is necessary for observing inhibition in the oculomotor system. To ensure that attention was maintained at the cued location, participants were asked to keep the location in visuospatial working memory (VSWM). Recent research into the mechanisms of VSWM has revealed a close link to the mechanisms involved in visual attention. The most prominent view is the attention-based rehearsal hypothesis (Awh, Vogel, \& Oh, 2006), according to which maintenance of spatial information in working memory is accomplished through a sustained shift of spatial attention to a memorized location. Evidence has come from a variety of psychophysical and neuroimaging studies showing enhanced visual processing at the location kept in memory (Awh, Anllo-Vento, \& Hillyard, 2000; Jha, 2002; Postle, Awh, Jonides, Smith, \& D'Esposito, 2004), analogous to the enhancement typically found during allocation of spatial attention.

A recent study (Theeuwes, Van der Stigchel, \& Olivers, 2006) showed that the magnitude of IOR was similar whether or not the cued location had to be kept in memory. It was suggested that IOR was caused merely by the exogenous activation of the oculomotor system through the transient activation caused by the abrupt onset of the cue. However, in this study, saccades were never made directly

\section{Experiment 1}

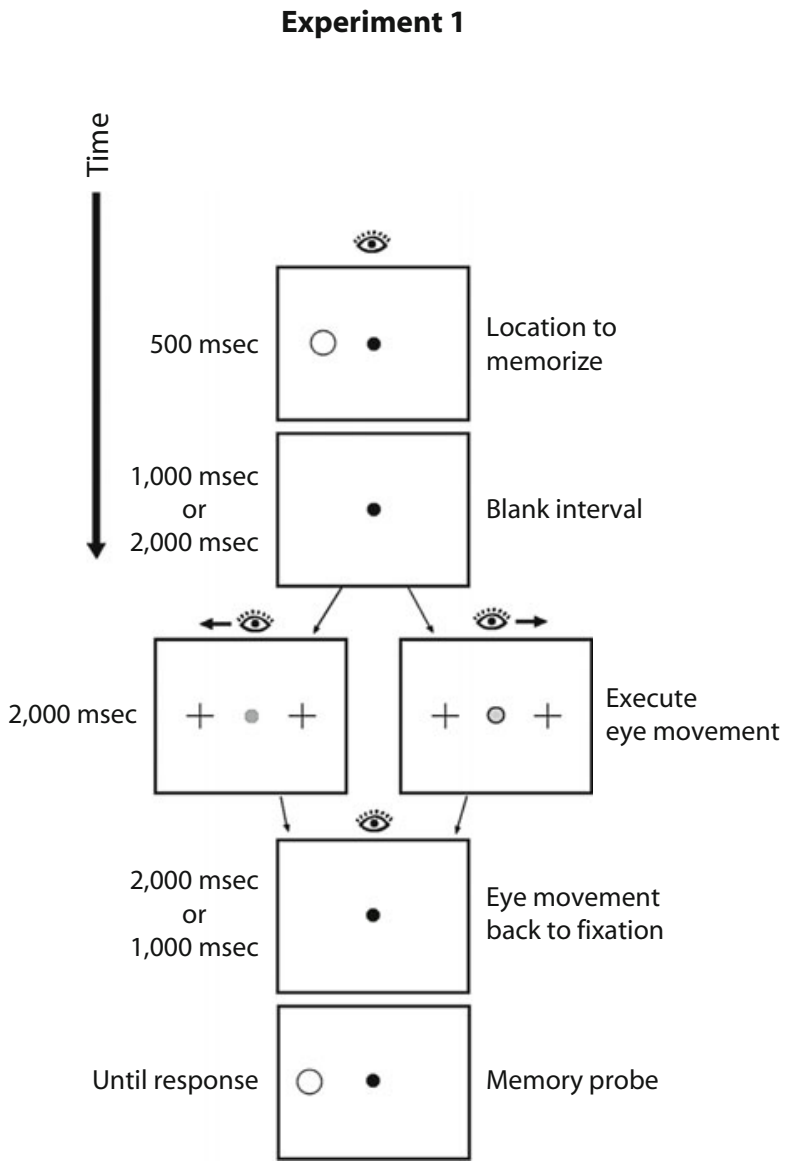

to the memorized location, which could have made it difficult to continuously maintain attention there. In addition, since a peripheral target was used to measure IOR, it remains unclear whether the oculomotor system was also inhibited.

To test whether disengagement of attention is necessary for producing oculomotor inhibition, on a proportion of trials, the participants were asked to saccade directly to the location kept in memory. If disengagement of attention is necessary, saccades should not be inhibited when participants are keeping the cued location in memory. In fact, given evidence for a obligatory coupling between attention and eye movements (Deubel \& Schneider, 1996), the reverse could be expected - that is, that saccades to the attended location are initiated more quickly than saccades to the unattended locations.

\section{EXPERIMENT 1}

In Experiment 1, participants were asked to maintain the location of a flashed circle in memory and to make a saccade in response to a central endogenous imperative signal (Figure 1, left panel). To estimate the effect of the maintenance of attention at the cued location on

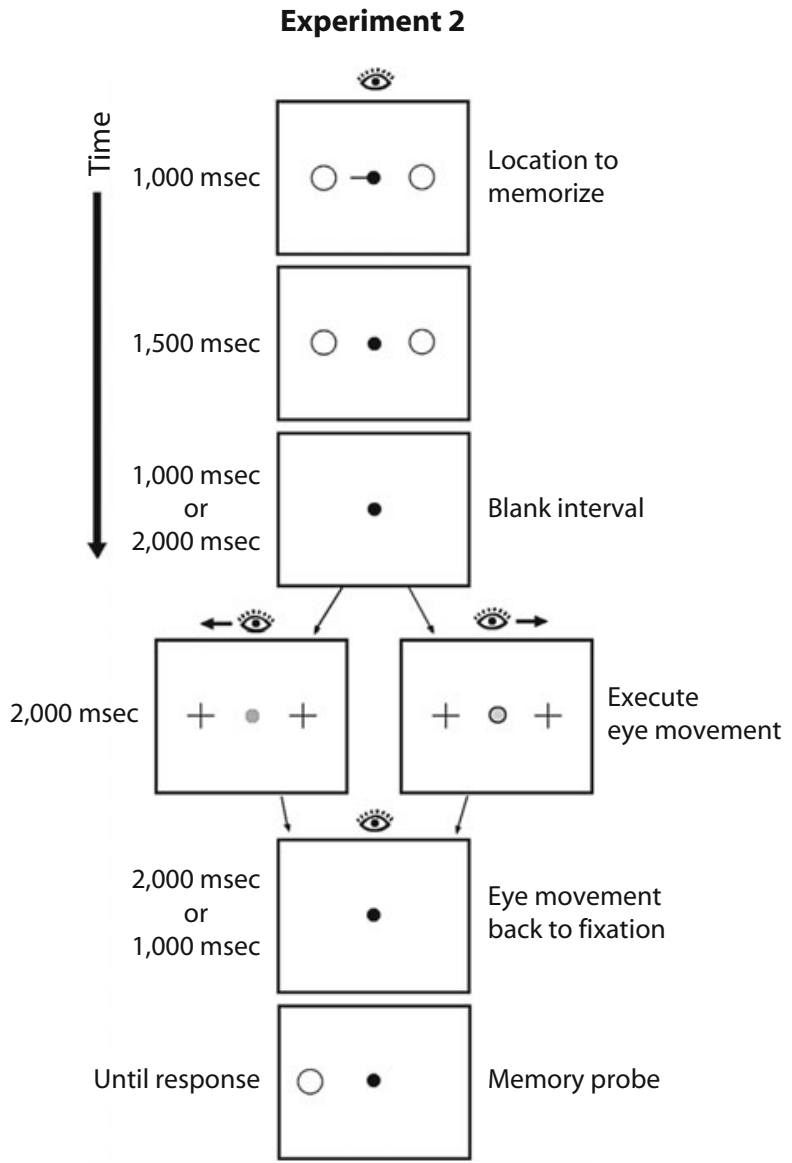

Figure 1. Typical task sequence. In Experiment 1 (left panel), the participants had to either memorize the location of the cue (memory condition) or ignore it (no-memory condition). In Experiment 2 (right panel), the participants had to memorize the location indicated by the arrow cue. 
oculomotor IOR, we included a no-memory condition, in which the participants did not have to keep the cued location in memory and, thus, did not have to maintain attention there.

\section{Method}

\section{Participants}

Sixteen volunteers from the Vrije Universiteit, with normal or corrected-to-normal vision, participated in the experiment and were randomly assigned to the memory and no-memory conditions. Memory and no-memory conditions were run between subjects to avoid carryover effects.

\section{Apparatus \\ A Pentium II computer with a 21 -in. color monitor generated the stimuli and controlled the timing of the events. Eye movements were recorded with an EyeLink tracker (500-Hz temporal and $0.2^{\circ}$ spatial resolution).}

\section{Stimuli, Design, and Procedure}

The stimuli were presented on a light gray background $\left(19.5 \mathrm{~cd} / \mathrm{m}^{2}\right)$. The trial began with the participants fixating the dot $\left(0.5^{\circ}\right.$ in diameter) at the center of the screen and pressing a space bar to start. After $500 \mathrm{msec}$, an exogenous memory cue (gray unfilled circle $1.35^{\circ}$ in diameter, $2.8 \mathrm{~cd} / \mathrm{m}^{2}$ ) appeared for $500 \mathrm{msec}$. In the memory condition, the participants had to memorize the exact location of the cue for $5,000 \mathrm{msec}$. The cue was equally likely to appear at any of the six possible locations on the horizontal axis: $4^{\circ}, 4.8^{\circ}$, and $5.6^{\circ}$ to the left and right of the fixation. To minimize the likelihood of verbal coding, we chose partially overlapping locations. On half of the trials, after a variable delay $(1,000$ or $2,000 \mathrm{msec}$ after cue offset), the fixation point changed color, and simultaneously two location markers $\left(2^{\circ}\right)$ were presented to the left and to the right of fixation. When green $\left(3.2 \mathrm{~cd} / \mathrm{m}^{2}\right)$, the fixation point indicated that an eye movement to the right location had to be made; when red $\left(3.2 \mathrm{~cd} / \mathrm{m}^{2}\right)$, it indicated that an eye movement to the left location had to be made. On half of these saccade trials, saccade goal coincided with the memorized location, whereas on the other half, a saccade had to be made to the equidistant location in the opposite hemifield (Figure 1, left panel). After 2,000 msec, the saccade markers disappeared, and the fixation point turned gray. At the end of the retention interval, a memory probe (identical to the cue) was presented either at the memorized location (probability of .5) or at a different location (shifted $0.7^{\circ}, 1.2^{\circ}$, or $1.7^{\circ}$ along the horizontal axis either toward or away from fixation). The direction and distance of the memory probe offset was selected randomly. The participants indicated whether the memory probe location was the same (" $m$ " key) or different ("v" key).

The participants were seated $75 \mathrm{~cm}$ from a computer screen. They received a block of 24 trials with the memory task alone, followed by a practice block of the saccade task alone ( 24 trials), in which the participants were instructed to make a saccade on the basis of the color of the fixation point. Practice blocks were followed by six experimental dual-task blocks ( 48 trials each), for a total of 288 trials. After each block, the participants received feedback about their average memory accuracy and saccade latency. On each trial, the participants received auditory feedback for memory and eye movement errors.

The no-memory condition was identical to the memory condition, except that the location of the exogenous cue did not need to be memorized. Therefore, in the no-memory condition, the last display containing the memory probe was omitted, as well as the memory practice block.

\section{Results}

Trials with saccades faster than $80 \mathrm{msec}$ and slower than $800 \mathrm{msec}$, saccades that did not start within $1^{\circ}$ of the
Table 1

Mean Saccade Direction Errors (\%) in Experiments 1 and 2

$\frac{1,000-m s e c \text { Delay }}{\text { Cued Uncued }} \quad \frac{2,000-m s e c \text { Delay }}{\text { Cued Uncued }}$

Location Location Location Location

\begin{tabular}{lrrrr}
\hline Experiment 1 & & & & \\
$\quad$ Memory condition & 5.8 & 6.6 & 6.5 & 7.0 \\
No-memory condition & 16.9 & 9.6 & 18.5 & 10.6 \\
Experiment 2 & 11.9 & 6.9 & 12.8 & 6.9 \\
\hline
\end{tabular}

fixation point, and trials with saccades to the cue were discarded from further analyses. This resulted in an average loss of $15 \%$ of the trials in the memory condition and $9 \%$ in the no-memory condition. Three participants in the memory condition and 1 participant in the no-memory condition were replaced due to excessive saccade direction errors $(>25 \%)$.

\section{Saccade Responses}

The participants made, on average, $6.5 \%$ saccade direction errors in the memory condition and $14 \%$ in the nomemory condition (Table 1). In the no-memory condition, there were more saccade direction errors when the saccade had to be made toward the cued location than when it had to be made away from the cued location $[F(1,7)=18.11$, $p<.005]$. No other effects were significant (all $F_{\mathrm{S}}<1$ ). These results are consistent with IORs being applied to the location of the cue and biasing saccades away from the inhibited location (Posner et al., 1985). No significant difference in the number of errors across conditions was found in the memory condition (all $F \mathrm{~s}<1$ ). The absence of an effect of making more saccade errors when a saccade toward the memorized location was required might suggest that there was some interaction between IOR and the process of maintaining location in memory. However, this biasing effect on errors is not consistently replicated in the literature, and it is not clear how much weight can be placed on it (see, e.g., Abrams \& Dobkin, 1994).

Mean correct saccade latencies are presented in Figure 2. To examine whether IOR was modulated by maintaining the location of the cue in memory, we conducted a mixed-effects ANOVA, with condition (memory or no memory) as the between-subjects factor and saccade delay $(1,000$ or 2,000 msec) and saccade target (cued or uncued) as the within-subjects factors. There was a main effect of condition, showing that adding a secondary memory task increased overall latencies $[F(1,14)=7.60, p<.05]$. Surprisingly, in both conditions, saccades were initiated more slowly toward the cued location, resulting in a main effect of saccade target $[F(1,14)=17.12, p<.005]$. Importantly, the three-way interaction was also significant $[F(1,14)=6.65, p<.05]$, suggesting that inhibition had a different time course across the conditions. Post hoc comparisons showed that at $1,000 \mathrm{msec}$, inhibition was not significantly different between the memory and nomemory conditions ( 4 vs. $10 \mathrm{msec}$, respectively; $F<1$ ). However, the inhibition was greater in the memory condition at 2,000 msec [ $22 \mathrm{vs} .6 \mathrm{msec}$, respectively; $F(1,14)=$ $5.73, p<.05]$. These results suggest that the oculomotor 


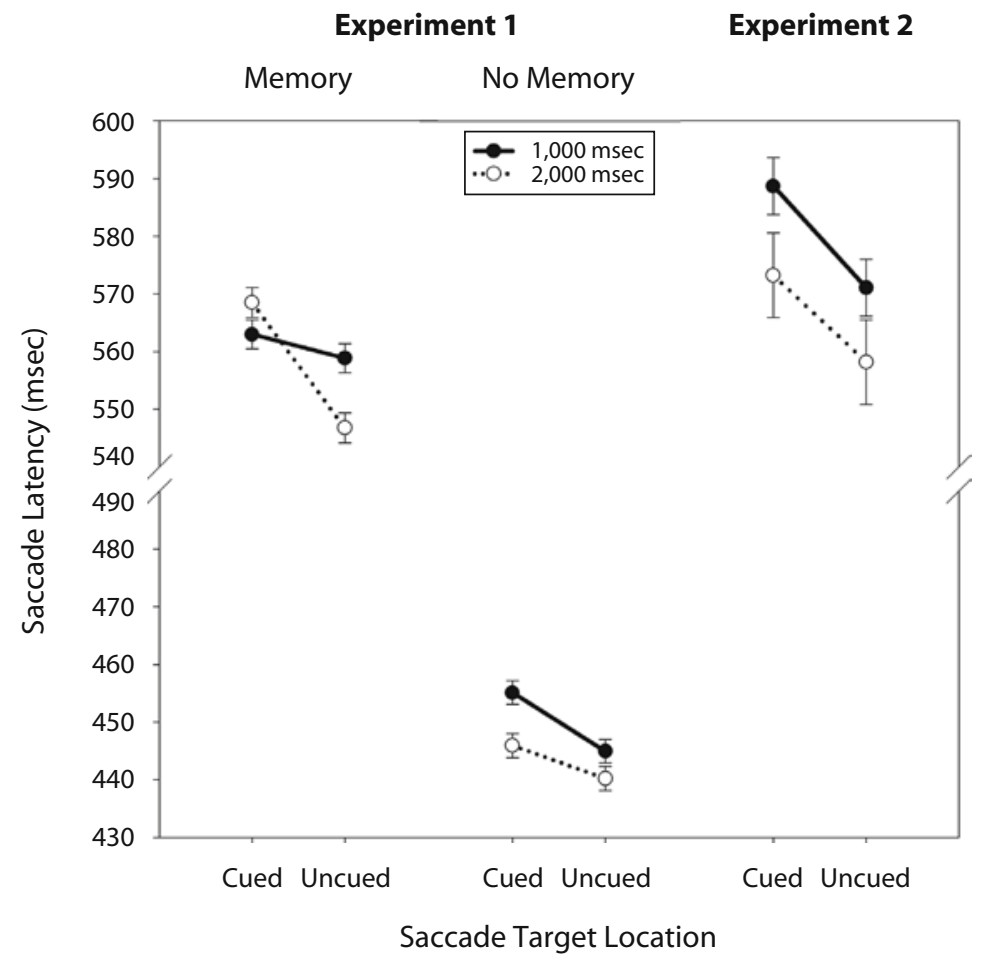

Figure 2. Mean correct saccade latencies in Experiments 1 and 2 as a function of saccade target location and delay. The error bars represent the normalized standard errors of the means for the saccade target location factor (Loftus \& Masson, 1994).

inhibition is observed even when the cued location has to be maintained in memory and its magnitude is increased at the longer delays.

\section{Memory Performance}

On average, the participants were quite accurate in the memory task (83\%). When the memory probe did not fall on the memorized location, the accuracy was dependent on the distance of the memory probe from the memorized location. Basically, the closer the memory probe was to the memorized location, the more difficult it was to detect the difference and to respond correctly [44\%,79\%, and $97 \%$ for close, medium, and far memory probe locations, respectively; $F(2,14)=93.01, p<.001]$. This suggests that the participants were maintaining a spatial code for the memorized location.

Planned comparisons showed that memory performance was not impaired when a saccade was made during the retention interval, as compared with trials on which no saccade was made (see Table 2). Whereas for the 1,000-msec delay, memory performance was not different between saccade and no-saccade trials $[82.6 \%$ vs. $81.0 \%$, respectively; $t(7)=0.72, p=.45]$, for the 2,000 -msec delay, there was a trend for the memory performance to be better during the saccade trials $[82.4 \%$ vs. $81.0 \% ; t(7)=2.1$, $p=.07]$. Memory performance for the saccade trials was submitted to a two-way repeated measures ANOVA with delay $(1,000$ or 2,000 $\mathrm{msec})$ and saccade target hemifield (cued or uncued) as factors. There were no significant dif- ferences in spatial memory performance across saccade delays or saccade target locations (all $F \mathrm{~s}<1.5$ ).

\section{Discussion}

In Experiment 1, we found a classic oculomotor IOR effect (Abrams \& Dobkin, 1994), despite the fact that location had to be kept in memory and endogenous attention was presumably maintained at the memorized location. Since we used an abrupt onset cue to indicate the location to be kept in memory, it is feasible that this oculomotor inhibitory effect was due to the direct exogenous activation of the oculomotor program (Berlucchi, Chelazzi, \& Tassinari, 2000; Rafal et al., 1989; Theeuwes et al., 2006). However, at the longer delays, oculomotor inhibition was stronger when the location was maintained in memory. Perhaps the oculomotor inhibition was at least partially caused by the oculomotor programming associated with the endogenous covert attention maintained at the memorized location. This alternative was tested in Experiment 2.

Table 2

Mean Correct Memory Responses (\%) in Experiments 1 and 2

\begin{tabular}{|c|c|c|c|c|c|}
\hline & \multicolumn{2}{|c|}{ 1,000-msec Delay } & \multicolumn{2}{|c|}{ 2,000-msec Delay } & \multirow[b]{2}{*}{$\begin{array}{c}\text { No } \\
\text { Saccade }\end{array}$} \\
\hline & $\begin{array}{c}\text { Cued } \\
\text { Location }\end{array}$ & $\begin{array}{c}\text { Uncued } \\
\text { Location }\end{array}$ & $\begin{array}{c}\text { Cued } \\
\text { Location }\end{array}$ & $\begin{array}{c}\text { Uncued } \\
\text { Location }\end{array}$ & \\
\hline Experiment 1 & 84.9 & 80.2 & 81.0 & 83.8 & 81.0 \\
\hline Experiment 2 & 77.1 & 81.0 & 77.4 & 74.1 & 75.9 \\
\hline
\end{tabular}




\section{EXPERIMENT 2}

To test whether the oculomotor inhibition found in Experiment 1 was caused by the oculomotor programming associated with keeping a location in memory, we used an endogenous cue to indicate the to-be-memorized location in Experiment 2. As was mentioned earlier, IOR is not typically observed with endogenous cues, unless they are instructing participants to prepare an eye movement (Rafal et al., 1989).

\section{Method}

Eight volunteers from the Vrije Universiteit, with normal or corrected-to-normal vision, participated in the experiment. The experiment was exactly the same as Experiment 1, except that an endogenous memory cue was used. The trial began with the presentation of one cue in each hemifield, and a central arrow indicated which cue had to be memorized. After $1,000 \mathrm{msec}$, the arrow disappeared, and after another 1,500 msec, both circles disappeared (Figure 1, right panel). This longer time course was chosen because endogenous orienting is known to be much slower than exogenous orienting (Cheal \& Lyon, 1991).

\section{Results}

Trials with saccades faster than $80 \mathrm{msec}$ and slower than $850 \mathrm{msec}$, saccades that did not start within $1^{\circ}$ of the fixation point, and trials with saccades to the cue were discarded from further analyses. This resulted in an average loss of $13 \%$ of the trials. Two participants were replaced due to excessive saccade direction errors $(>25 \%)$.

\section{Saccade Responses}

The participants made, on average, $10 \%$ saccade direction errors (Table 1), and there was a trend to make more errors when saccade had to be made to the cued location $[F(1,7)=4.28, p=.08]$. No other effects were significant (all $F \mathbf{s}<1)$. The participants were faster in making a saccade after a longer delay $[F(1,7)=6.73, p<.05$; see Figure 2]. Importantly, the participants were significantly slower in making a saccade toward the memorized location $[F(1,7)=8.86, p<.05]$. The interaction was not significant $(F<1)$. These results suggest that oculomotor inhibition is present even when the memorized location is indicated in an endogenous fashion. Therefore, it is the process of keeping the location in memory, and not the nature of the memory cue, that already engages the oculomotor system.

\section{Memory Performance}

On average, the participants were quite accurate in the memory task $(76 \%)$. When the probe did not coincide with the memorized location, the closer it was to the memorized location, the more difficult it was to detect the difference and to respond correctly $[31 \%, 75 \%$, and $82 \%$ for close, medium, and far memory probe locations, respectively; $F(2,14)=102.0, p<.001]$.

Planned comparisons showed that memory performance was not impaired when a saccade was made during the retention interval, as compared with trials on which no saccade was made (see Table 2). For both the 1,000msec $(79 \%)$ and 2,000-msec (76\%) delay, memory perfor- mance was not different from that on the no-saccade trials $[76 \% ; t(1,7)=1.75, p=.12$, and $t<0.5$, respectively]. Memory performance on the saccade trials was submitted to two-way repeated measures ANOVA with delay $(1,000$ or 2,000 msec) and saccade target hemifield (cued or uncued) as factors. There were no significant differences in spatial memory performance across saccade delays or saccade target locations [for main effects, $F \mathbf{s}<1$; for the interaction, $F(1,7)=2.41, p=.16]$.

\section{Discussion}

The present results clearly show that inhibition of saccades to the location kept in spatial working memory is due to its maintenance in working memory and not to whether it is cued in exogenous or endogenous fashion. Therefore, it appeared that maintaining attention at the memorized location coexisted with inhibition of saccades to that location. However, before such a conclusion can be drawn, two possible concerns have to be addressed. First of all, we have assumed that spatial attention is allocated to the memorized location on the basis of the large number of previous studies that have demonstrated facilitation of visual processing at the memorized location during the retention interval (Awh et al., 2000; Awh, Jonides, \& Reuter-Lorenz, 1998; Jha, 2002). Although the spatial memory task that we used was very similar to the one used in these studies, there might have been an important difference.

Specifically, we used a rather restricted set of possible memory locations (only six locations; note, however, that this is the same number as in Jha, 2002), which might have discouraged the participants from maintaining a precise memory code of the cued location. It is possible that, as a consequence, a sharp focus of attention was not maintained at the memorized location. One could argue that if a larger number of possible memory locations had been used, that would have forced the participants to have a tighter attentional focus at the memorized location and could have led to facilitation of oculomotor responses.

Another concern is that several previous studies (Baddeley \& Lieberman, 1980; Lawrence, Myerson, \& Abrams, 2004; Lawrence, Myerson, Oonk, \& Abrams, 2001) have demonstrated that making a saccade during the retention interval caused impairment in the accuracy of the spatial memory representation, as compared with when no saccade was required. However, no such impairment was found in Experiments 1 and 2. The absence of memory impairment in these experiments could also potentially stem from a coarse spatial memory coding, given a small number of possible memory locations. These two concerns regarding the maintenance of endogenous attention at the memorized location and the interference of a saccade task with the spatial working memory task were addressed in Experiment 3.

\section{EXPERIMENT 3}

The goal of Experiment 3 was to provide additional evidence that endogenous attention is maintained at the memorized location throughout the retention interval and can 
coexist with inhibition of saccades to that location. As in Experiment 2, an endogenous memory cue was used, but there were 84 possible memory cue locations (42 locations in each hemifield). In Experiment 3A, just as in the previous experiments, the participants had to make a saccade to the left or right location, depending on the cue at the fixation. If inhibition of saccades to the memorized location was due to imprecise memory coding, no inhibition should be found in the present experiment. In addition, Experiment 3B used a very similar setup, but the participants had to maintain fixation at the center throughout the whole trial and manually respond to a target presented during the retention interval. If attention is maintained at the memorized location during the retention interval, manual responses should be faster when the target occurs at the memorized location, as compared with the other locations.

A closer look at the design of Experiments 1 and 2 shows that the absence of interference between the saccade task and the spatial working memory task is not that surprising. Note that in both experiments, on saccade trials, a placeholder was always presented at the memorized location, independently of where the saccade had to be made. Therefore, the lack of memory impairment, relative to no-saccade trials, could have been due to the simple fact that, on saccade trials, the memorized location was always stimulated. To examine this in Experiment 3, we included a condition in which no placeholder was presented at the memorized location when a saccade had to be made during the retention interval. If stimulation of the memorized location was responsible for recovering memory accuracy on saccade trials, memory should be significantly impaired when such a stimulation does not occur.

\section{Participants}

Twenty volunteers from the Vrije Universiteit, with normal or corrected-to-normal vision, participated in the experiment and were randomly assigned to Experiments $3 \mathrm{~A}$ (saccade response, 10 participants) and 3B (manual response, 10 participants). Two experiments were run between subjects to avoid carryover effects.

\section{Stimuli, Design, and Procedure}

In Experiment 3A, the participants had to make an eye movement in response to the color change at the fixation, and in Experiment 3B, the participants had to make a manual response to a target. In general, the setup of Experiment $3 \mathrm{~A}$ was very similar to that in Experiment 2 (see Figure 3). The biggest difference was that there were 84 possible memory cue locations, with 28 equally spaced lo-

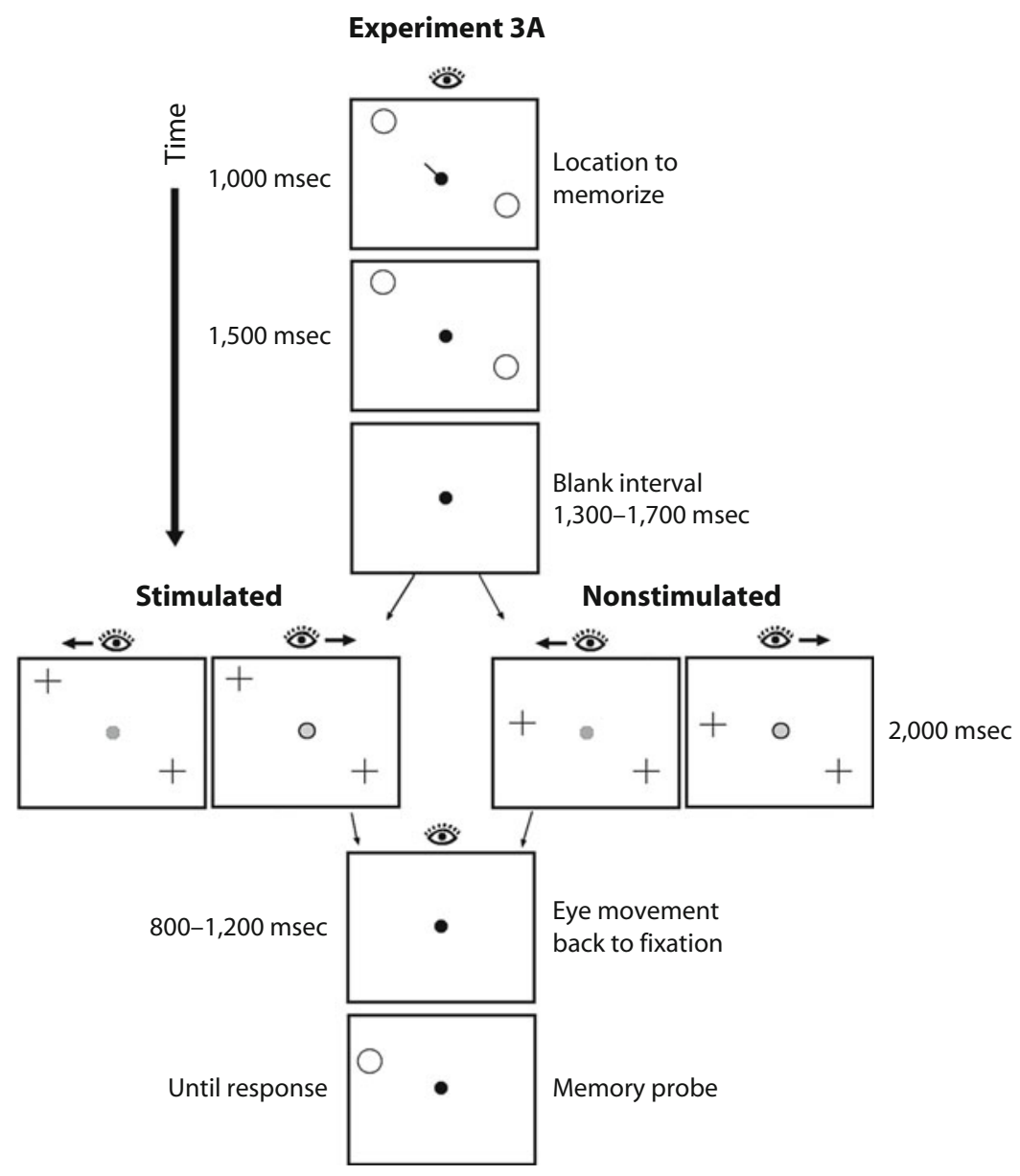

Figure 3. Typical task sequence in Experiment 3A. In Experiment 3B, instead of the two "+" signs, a "+" and an "x" were presented simultaneously. A "+" indicated the target, and an " $x$ " indicated a distractor. 


\section{Experiment 3A}

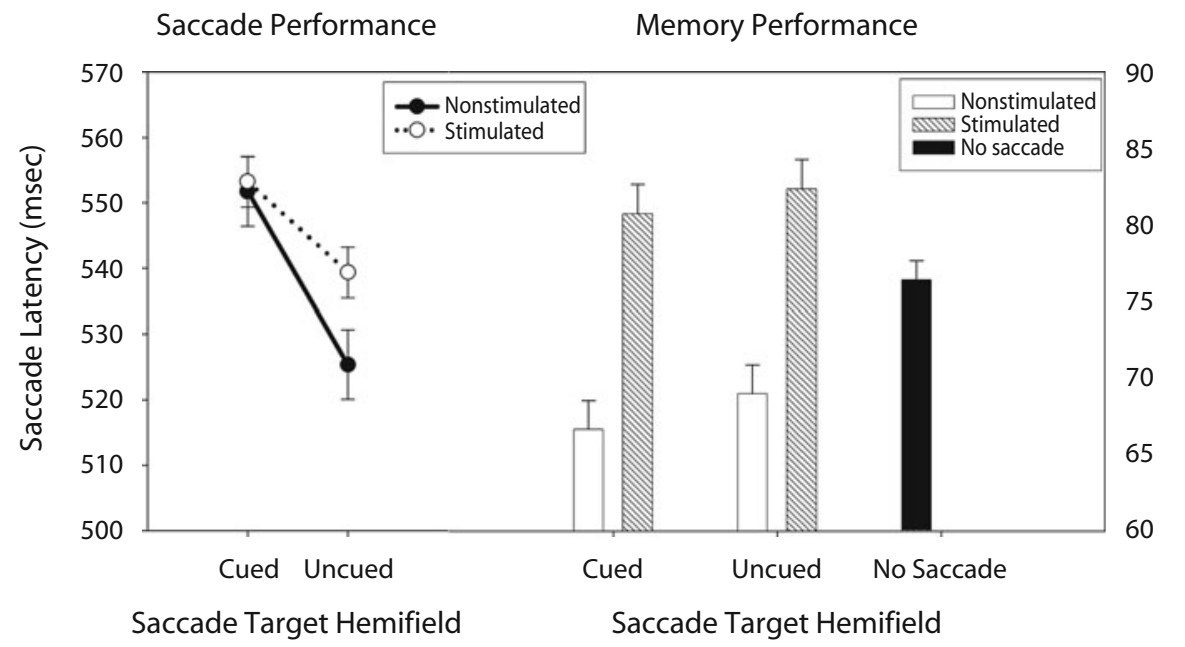

Figure 4. Left panel: Mean correct saccade latency in Experiment 3A as a function of saccade target hemifield and delay. Right panel: Mean memory accuracy as a function of saccade presence, saccade target hemifield, and delay. The error bars represent the normalized standard errors of the means for the saccade hemifield factor (Loftus \& Masson, 1994). For the no-saccade trials, the error bars represent nonnormalized standard errors of the means.

cations (each $1^{\circ}$ in diameter) on each of the three imaginary circles (radius of $4^{\circ}, 4.8^{\circ}$, or $5.6^{\circ}$ ), excluding the locations falling on all cardinal axes (4 locations) and the locations neighboring the vertical axes (4 locations). As in Experiment 2, the trial began with the presentation of one cue in each hemifield, and a central arrow indicated which location had to be memorized. The locations of the cues were randomly chosen within each hemifield, with the constraint that both locations always fell on the same circle. Memorized location was equally likely to appear in the left or the right hemifield.

On half of the trials, after a variable delay (randomized between 1,300 and 1,700 msec after cue offset), the fixation point changed color, and simultaneously two location markers $\left(1.2^{\circ}\right)$ were presented to the left and to the right of the fixation. Eye movement was equally likely to be made to the left and to the right hemifield. To control for the effect of stimulation of the memorized location, one of the location markers was presented at the memorized location on half of the trials. On the other half of the trials, the location marker was presented at another randomly chosen location in the same hemifield and on the same imaginary circle as the memorized location and had to be at least two positions away from it.

Therefore, on half of the saccade trials, the saccade goal was presented in the same hemifield as the memorized location, whereas on the other half, a saccade had to be made to the equidistant location in the opposite hemifield. At the same time, the memorized location either could be stimulated by the location marker appearing at its location or could be left nonstimulated, with the location marker appearing in the same hemifield as the memorized location. Thus, the factors of saccade target hemifield (cued or uncued) and stimulation of memorized location (stimulated or nonstimulated) were manipulated orthogonally (Figure 3). Location markers were presented for $2,000 \mathrm{msec}$. The duration of the retention interval was kept constant at $4,500 \mathrm{msec}$. The rest of the design and procedure was identical to that of Experiment 2.

In Experiment 3B, a setup that was exactly the same as that in Experiment $3 \mathrm{~A}$ was used, but instead of making a saccade, the participants had to maintain fixation at the center and detect a target by pressing a space bar. The target was always a "+" sign, and the distractor was always an " $x$ "; both were simultaneously present in the display. To make the task as similar as possible to the saccade task used in Experiment $3 \mathrm{~A}$, the participants were asked to respond to the target only if it occurred in the hemifield indicated by the color of the fixation point (go trials). Therefore, the participants had to respond to the target on the right only if the fixation point was green and to refrain from responding if the fixation point was red. Analogously, the participants had to respond to the target on the left only if the fixation point was red and to refrain from responding if the fixation point was green. Trials on which the participants had to withhold their response (no-go trials) were presented on one third of the trials.

\section{Results}

\section{Experiment 3A}

Trials with saccades faster than $80 \mathrm{msec}$ and slower than $850 \mathrm{msec}$, saccades that did not start within $1^{\circ}$ of the fixation point, and trials with saccades to the cue were discarded from further analyses. This resulted in an average loss of $24 \%$ of the trials. One participant was replaced because of excessive saccades to the memory cue $(>15 \%)$. One participant was replaced due to excessive saccade direction errors $(>25 \%)$. Memory performance and saccade responses are presented in Figure 4.

Saccade responses. The participants made, on average, $14 \%$ saccade direction errors (Table 3). Saccade direction errors and saccade latencies were submitted to the two-way repeated measures ANOVA with stimulation (memory location stimulated or not) and saccade target

Table 3

Mean Saccade Direction and

Manual Errors (\%) in Experiment 3

\begin{tabular}{cccccc}
\hline \multicolumn{3}{c}{ Manual Errors (\%) in Experiment 3 } \\
& \multicolumn{2}{c}{$\begin{array}{c}\text { Nonstimulated } \\
\text { Memory Location }\end{array}$} & & \multicolumn{2}{c}{$\begin{array}{c}\text { Stimulated } \\
\text { Memory Location }\end{array}$} \\
\cline { 2 - 3 } \cline { 5 - 6 } & $\begin{array}{c}\text { Cued } \\
\text { Hemifield }\end{array}$ & $\begin{array}{c}\text { Uncued } \\
\text { Hemifield }\end{array}$ & & $\begin{array}{c}\text { Cued } \\
\text { Hemifield }\end{array}$ & $\begin{array}{c}\text { Uncued } \\
\text { Hemifield }\end{array}$ \\
\hline Experiment 3A & 19.0 & 13.0 & & 16.0 & 9.0 \\
Experiment 3B & 1.3 & 2.9 & & 4.4 & 3.1 \\
\hline
\end{tabular}


hemifield (cued or uncued) as factors. The participants made more errors when the memorized location was not stimulated $[16 \%$ vs. $13 \% ; F(1,9)=5.34, p<.05]$. Importantly, more errors were also made when a saccade had to be made to the target in the same hemifield as the memorized location $[18 \%$ vs. $11 \% ; F(1,9)=7.37, p<.05]$. The interaction was not significant $(F<1)$. Similarly, the participants were significantly slower in executing a saccade toward the hemifield containing the memorized location [552 vs. $532 \mathrm{msec} ; F(1,9)=6.20, p<.05]$. The effect of stimulation on saccadic latencies was not significant $[F(1,9)=2.75, p=.13]$. The interaction also was not significant $[F(1,9)=1.87, p=.21]$. Taken together, the results from both saccadic latencies and direction errors suggest that initiation of saccades to the hemifield containing the memorized location was suppressed.

Memory performance. On average, the participants were quite accurate in the memory task (74.6\%). When the probe did not coincide with the memorized location, the closer it was to the memorized location, the more difficult it was to detect the difference and to respond correctly $[38 \%, 62 \%$, and $92 \%$ for close, medium, and far memory probe locations, respectively; $F(2,18)=61.82$, $p<.001]$.

Planned comparisons showed that memory performance was impaired when a saccade was made and the memorized location was not stimulated, as compared with trials on which no saccade had to be made [68\% vs. $76 \%$ correct, respectively; $t(9)=3.46, p<.01]$. When saccades were made but memory location was stimulated, memory performance was actually better than that in the no-saccade condition $[82 \%$ vs. $76 \%$, respectively; $t(9)=$ $2.45, p<.05]$. These results suggest that stimulation of memory location during the retention interval not only helps to overcome the negative effect of making a saccade away from the memorized location, but also improves memory performance. Planned comparison also showed that making a saccade to the stimulated memory location does not result in an additional increase in memory performance, relative to when a saccade away from the stimulated memory location has to be made ( $81 \%$ vs. $82 \%$, respectively; $t<1$ ).

Memory performance for the saccade trials was submitted to a two-way repeated measures ANOVA with stimulation (memory location stimulated or not) and saccade target hemifield (cued or uncued) as factors. There was a main effect of stimulation $[F(1,9)=32.0, p<.001]$, with stimulated locations remembered better than nonstimulated locations. No other effects were significant $(F<1)$.

\section{Experiment 3B}

One participant was replaced because of a high error rate $(>10 \%)$. Go trials on which the participants made eye movements $(<5 \%)$ and trials on which the participants responded more quickly than $150 \mathrm{msec}$ or more slowly than $1,800 \mathrm{msec}(<5 \%)$ were discarded from the analysis. Memory performance and manual reaction times are presented in Figure 5.

Manual responses. The go/no-go task was quite difficult, and the overall reaction times were relatively long. Nevertheless, the participants were faster in detecting the target in the hemifield containing the memorized location [947 vs. $1,011 \mathrm{msec} ; F(1,9)=13.87, p<.01]$. They were also overall faster to respond to the target when the memorized location was stimulated $[F(1,9)=6.76, p<.05]$, suggesting that presenting a stimulus at a nonmemorized location interfered with target detection. The interaction was not significant $(F<1)$. The results suggest that visual processing of targets in the hemifield containing the

\section{Experiment 3B}

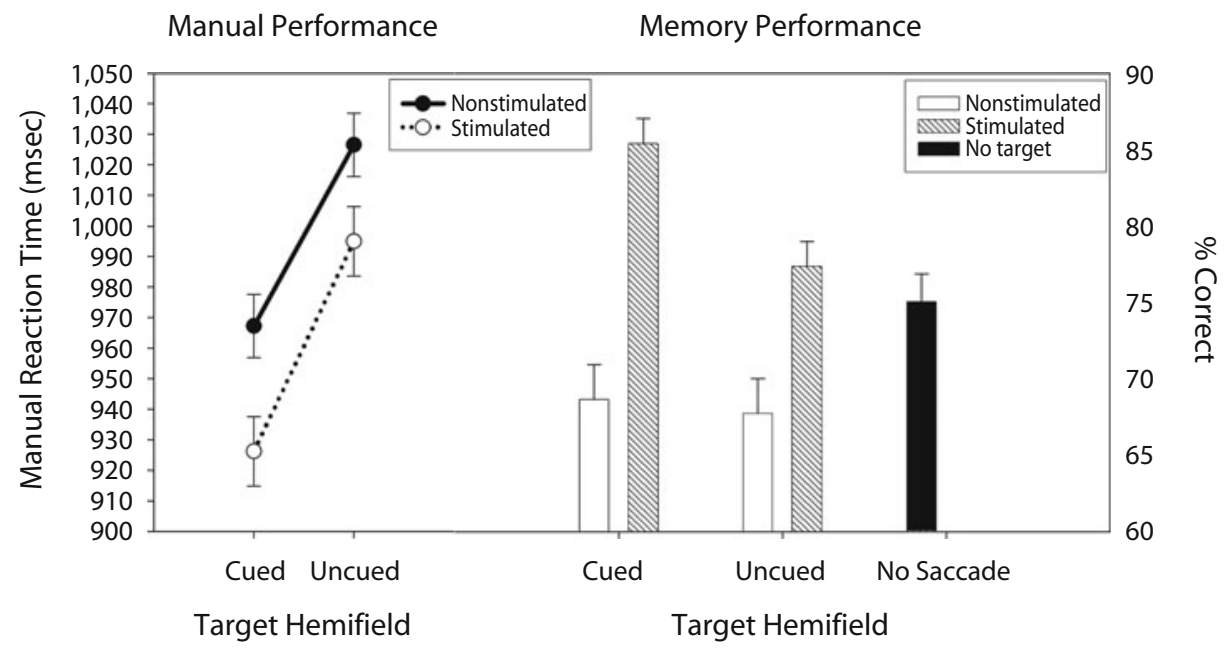

Figure 5. Left panel: Mean correct manual reaction times in Experiment 3B as a function of target hemifield and delay. Right panel: Mean memory accuracy as a function of target presence, target hemifield, and delay. The error bars represent the normalized standard errors of the means for the target hemifield factor (Loftus \& Masson, 1994). For the no-target trials, the error bars represent nonnormalized standard errors of the means. 
memorized location is facilitated. The participants made, on average, $3 \%$ errors in target detection (Table 3 ). Main effects and the interaction were not significant.

The false alarm rate was low (13\%). The participants made more false alarms when the memorized location was stimulated than when it was not stimulated $[17 \%$ vs. $10 \%$, respectively; $F(1,9)=6.24, p<.05]$. This suggests that responses to stimuli occurring at the location kept in memory were more difficult to suppress than responses to stimuli occurring at other locations. Other effects and the interaction were not significant.

Memory performance. On average, the participants were quite accurate in the memory task (74.4\%). When the probe did not coincide with the memorized location, the closer it was to the memorized location, the more difficult it was to detect the difference and to respond correctly [35\%, 66\% and $83 \%$ for close, medium, and far memory probe locations, respectively; $F(2,18)=56.44, p<.001$ ]

Planned comparisons showed that responding to the target impaired memory performance when the memorized location was not stimulated, as compared with trials on which no stimuli were presented during the retention interval $[68 \%$ vs. $75 \%$ correct, respectively; $t(9)=2.32$, $p<.05]$. This means that attentional shifts during the retention interval interfered with memory maintenance. However, when the memory location was stimulated, memory performance was marginally better than in the no-target condition $[81 \%$ vs. $75 \%$, respectively; $t(9)=$ $2.11, p=.06]$. These results suggest that stimulation of memory location during the retention interval not only helps to overcome the negative effect of shifting attention away from the memorized location, but also tends to improve memory accuracy. Planned comparison also showed that keeping attention at the stimulated memory location results in an additional increase in memory performance, relative to when attention has to be shifted away from the stimulated memory location $[85 \%$ vs. $77 \%$, respectively; $t(9)=2.44, p<.05]$.

Memory performance for the target-present trials was also submitted to a two-way repeated measures ANOVA with stimulation (memory location stimulated or not) and target hemifield (cued or uncued) as factors. There was a main effect of stimulation $[F(1,9)=30.86, p<.001]$, with stimulated locations remembered better than nonstimulated locations. Neither the effect of target hemifield $[F(1,9)=2.38, p=.16]$ nor the interaction $[F(1,9)=1.7$, $p=.23$ ] was significant.

\section{Discussion}

The results of Experiment 3 clearly dismiss the two concerns that arose from Experiments 1 and 2. First, increasing the number of possible memory locations from 6 to 84 led to basically the same results for the saccade performance (Experiment 3A): Saccades directed to the location that was in the same hemifield as the location maintained in memory were initiated more slowly than saccades to the opposite hemifield. There was also a decrease in accuracy when saccades were required to the same hemifield as that of the location maintained in memory. Taken together, these results suggest that saccades were biased away from the location maintained in the spatial working memory and that this bias extended to the whole visual hemifield. Therefore, it was unlikely that inhibition of saccades to a location kept in memory was due to the imprecise memory coding and the lack of focused attention at the memorized location.

Experiment 3B provided additional evidence that endogenous attention was maintained at the memorized location. It used a design that was very similar to that in Experiment 3B, but instead of a saccade, a manual response was required. Consistent with previous studies (Awh et al., 2000; Awh et al., 1998; Jha, 2002), targets presented at the memorized location were detected more quickly than targets at the nonmemorized locations, suggesting that visual processing at the memorized location was enhanced.

Second, Experiment 3 showed that both eye movements and attentional shifts interfered with the maintenance of spatial working memory (Baddeley \& Lieberman, 1980; Lawrence et al., 2004; Lawrence et al., 2001). This impairment was observed only when the memorized location was not stimulated during the retention interval. In both Experiments $3 \mathrm{~A}$ and $3 \mathrm{~B}$, when a stimulus was presented at the memorized location, memory for that location was refreshed, resulting not only in compensation for making a saccade or shifting attention, but also, in most cases, in improvement in memory performance. Therefore, the lack of interference between saccades and spatial working memory maintenance observed in Experiments 1 and 2 may have been due to the stimulation of the memorized location during the retention interval.

Interestingly, in Experiment 3A, just as in Experiments 1 and 2, making a saccade to the memorized location did not give any additional benefit to memory accuracy (Figure 4, right panel). This result, however, is not surprising, given that the memorized location was still being stimulated after the saccade had been made and the eyes had returned to fixation. Therefore, even when a saccade was made away from the memorized location, there was still time to attend to the stimulated memorized location, once the eyes returned to fixation. On the contrary, only manual detection of a target at the memorized location in Experiment 3B gave an additional boost to memory performance (Figure 5, right panel). Judging from reaction times, processing of the target up to the level of manual response took longer than making a saccade. Thus, when the target appeared at the nonmemorized location, there was less time available to return attention to the stimulated memorized location. Nevertheless, although shifting attention away from the stimulated memorized location did not result in an improvement in memory performance, it also did not lead to a decrease in memory performance, relative to the no-target condition. Whether this memory sparing is a result of passive stimulation of the memorized location or active allocation of attention to the stimulated location after an attentional shift away is completed requires further investigation. 


\section{GENERAL DISCUSSION}

The present findings suggest that disengagement of endogenous attention is not necessary to observe the oculomotor IOR. Consistent with previous studies, we found oculomotor IOR when attention did not have to be maintained at the cued location (Abrams \& Dobkin, 1994). However, even when the participants engaged attention at this location for later memory recall (Awh, Vogel, \& Oh, 2006), we observed oculomotor IOR; that is, saccades were slower to the cued (memorized) locations than to uncued locations. Importantly, as was shown in Experiment 1 , later in the retention interval, maintaining attention at the cued location was associated with even greater inhibition of saccade initiation. Inhibition was present even when the location that needed to be kept in memory was indicated endogenously (Experiment 2). The results extend previous findings by showing that it is keeping a location in memory, and not the nature of the memory cue, that causes oculomotor inhibition (Theeuwes et al., 2006). It was also demonstrated that oculomotor inhibition is not confined to the memorized location. Initiation of saccades toward other locations in the visual hemifield containing the memorized location was also delayed and associated with increased error rates (Experiment 3A). Importantly, it appears that oculomotor inhibition can coexist with enhanced visual processing at the memorized location (Experiment 3B). Just as in previous studies (Baddeley \& Lieberman, 1980; Lawrence et al., 2004; Lawrence et al., 2001), we found that making saccades and shifting attention interfered with the maintenance of spatial information in working memory, unless the memorized location was stimulated during the retention interval. However, with the results of the present study, we can go beyond the mere interference effects and examine the mechanisms involved in the maintenance of spatial working memory.

Given a tight coupling between attention and eye movements (Deubel \& Schneider, 1996; Hoffman \& Subramaniam, 1995; Kowler, Anderson, Dosher, \& Blaser, 1995; Shepherd, Findlay, \& Hockey, 1986), one could have expected saccades to be initiated faster when attention is already allocated to the location of a saccade target. In fact, if covert attention is nothing other than programming of an eye movement (as first suggested by Klein, 1980, and promoted by the premotor theory of attention; Rizzolatti, Riggio, Dascola, \& Umiltà, 1987), the maintenance of attention at a location should facilitate saccades to that location, given that the oculomotor program is already available. This issue is still highly debatable (see Awh, Armstrong, \& Moore, 2006), with some researchers arguing for a close interdependence of attention and eye movements and others arguing for independence (Hunt \& Kingstone, 2003; Klein, 1980; Klein \& Pontefract, 1994). In the present study, we consistently found inhibition of eye movements directed toward the location held in memory. One possibility is that maintaining covert visual attention at a location results in suppression of oculomotor orienting to that location.

A similar idea of oculomotor suppression taking place during covert attentional orienting was proposed by Ber- lucchi and colleagues (Berlucchi et al., 2000; Tassinari, Aglioti, Chelazzi, Marzi, \& Berlucchi, 1987). They suggested that reaction time inhibition at the exogenously cued location is caused by "suppression of a natural ocular reaction toward a lateralized cue in order to maintain fixation" (Berlucchi, 2006, p. 1072). For the endogenous shifts of attention, a similar result has been reported by Klein and Pontefract (1994; however, this was not replicated by Hunt \& Kingstone, 2003). In their study, participants had to orient attention to a location likely to contain a target required for the manual detection task. On $20 \%$ of the trials, they received an auditory cue instructing them to make a saccade to either the attended or the unattended location. It was found that saccades directed toward the endogenously attended location were initiated more slowly and less accurately than saccades toward the unattended locations.

The oculomotor suppression hypothesis can be extended to the endogenous shifts of attention associated with maintenance of VSWM. Instruction to memorize a location might require suppression of a natural tendency to make an eye movement to that location, especially if a saccade toward that location is not required on the majority of trials. It is important to note that in our task, the participants were never instructed to make a saccade in response to the memory cue, and the direction of the upcoming saccade was not known in advance. In fact, on half of the trials, no saccade had to be made. Therefore, it appears that it was the process of orienting of attention in service of a memory task that engaged the memorized location in the oculomotor system.

Recently, Berlucchi and colleagues (see also Berger, Henik, \& Rafal, 2005; Berlucchi et al., 2000; Lupiáñez et al., 2004) provided evidence that endogenous covert orienting to a location does not suppress inhibitory processing at that location. Using independent manipulation of endogenous and exogenous attention, they demonstrated that the benefits of endogenous attention were smaller at the inhibited location than at a location not affected by IOR. These results strongly argue against disengagement of attention from the cued location being the mechanism of IOR generation. Importantly, IOR of the same magnitude was present irrespective of whether endogenous attention was maintained at that location, suggesting that the effects of endogenous and exogenous attention could be independent from each other.

Note that the reorienting hypothesis of IOR can be saved if the model of attention as a single spotlight, which was originally proposed by Posner (1980), is abandoned. According to this relatively new position, "IOR may delay exogenous orienting based on bottom-up signals without affecting endogenous orienting" (Lupiáñez, Klein, \& Bartolomeo, 2006, p. 1006). Therefore, disengagement of exogenous attention could signal the start of IOR. It is also conceivable, however, that IOR begins with the appearance of the cue (see Klein, 2000, Box 1). Whether IOR starts with the removal of (exogenous) attention from the cued location or begins immediately with the onset of the cue remains one of the main outstanding theoretical questions (see "Outstanding Questions," Klein, 2000, p. 145). 
The present study has clearly demonstrated suppression of oculomotor responses toward the memorized location, where attention is allocated endogenously (Awh, Vogel, $\& \mathrm{Oh}, 2006)$. This is consistent with the idea of a dissociation between endogenous attention and oculomotor preparation. Since we have controlled for the exogenous nature of the memory cue, we can be sure that the observed oculomotor inhibition is different from the classic IOR. Despite the fact that the effects are different (one being caused by a peripheral onset and the other by an endogenous shift of attention), the underlying mechanisms could be similar. Both effects could stem from inhibition in the oculomotor system caused by a low probability of executing a saccade to the attended location.

However, in our view, the observed dissociation between endogenous attention and oculomotor preparation does not necessarily mean independence between endogenous attention and oculomotor preparation. The oculomotor system was suppressed, but it was not neutral in relationship to attentional allocation, as would be expected under the independence account. A relevant result has recently been reported by Van der Stigchel and Theeuwes (2007), who showed that saccades deviated away from the locations where endogenous attention was maintained. It was proposed that endogenous attention resulted in activation in the oculomotor system that had to be inhibited in order for an eye movement in a different direction to be made (see also Sheliga, Riggio, \& Rizzolatti, 1995). It appears that the oculomotor system is always affected by the distribution of endogenous attention, indicating that the two systems are interdependent and that affecting one system always has an influence on the other system.

Our results also have potential implications for the mechanisms of spatial working memory. It has been proposed that endogenous attention plays a functional role in the maintenance of spatial representations in working memory (Awh et al., 1998). The present findings suggest that spatial working memory also affects the oculomotor system. A close link between spatial working memory and the oculomotor system has recently been demonstrated by Theeuwes and colleagues (Theeuwes, Olivers, \& Chizk, 2005; Theeuwes et al., 2006). They showed that keeping a location in memory causes saccade trajectories to deviate away from that location, just as saccades deviate from the visually presented stimuli that need to be ignored (Sheliga, Riggio, \& Rizzolatti, 1994). In other words, there is accumulating evidence that the oculomotor system is used to code and maintain location information in memory (Theeuwes et al., 2005; Theeuwes et al., 2006).

Interestingly, IOR has also been implicated as playing a role in spatial working memory (Castel, Pratt, \& Craik, 2003; Klein, 2000), given its proposed role as a foraging facilitator (Klein, 2000). Recently, Castel et al. showed that IOR was eliminated when a spatial working memory load was introduced at the fixation but was still present with nonspatial working memory secondary tasks. Such selective disruption suggested that IOR was also relying on spatial working memory resources (Baddeley, 1986).

In summary, we have demonstrated that maintaining attention at the memorized location results in the suppres- sion of saccadic eye movements to that location. Therefore, disengagement of endogenous attention cannot be the mechanism responsible for inhibition of the oculomotor system. This oculomotor inhibition might be similar to IOR and could reflect a suppression of a natural tendency to make a saccade to a memorized location. In addition, we propose that the effects of endogenous attention and oculomotor programming can be dissociated, so that facilitation of sensory processing at a memorized location may coexist with suppression of oculomotor orienting to that location.

\section{AUTHOR NOTE}

This research was funded by grants from the Human Frontier Science Program (HSFP-RGP39/2005) to J.T. We thank Guido Peek for his help in data collection. We also thank Ray Klein, Joshua Solomon, and an anonymous reviewer for their comments. Correspondence concerning this article should be addressed to A. V. Belopolsky, Department of Cognitive Psychology, Vrije Universiteit, Van der Boechorststraat 1, 1081 BT Amsterdam, The Netherlands (e-mail: a.belopolsky@psy.vu.nl).

\section{REFERENCES}

Abrams, R. A., \& Dobkin, R. S. (1994). Inhibition of return: Effects of attentional cuing on eye-movement latencies. Journal of Experimental Psychology: Human Perception \& Performance, 20, 467-477.

Awh, E., Anllo-Vento, L., \& Hillyard, S. A. (2000). The role of spatial selective attention in working memory for locations: Evidence from event-related potentials. Journal of Cognitive Neuroscience, $\mathbf{1 2}$, 840-847.

Awh, E., Armstrong, K. M., \& Moore, T. (2006). Visual and oculomotor selection: Links, causes and implications for spatial attention. Trends in Cognitive Sciences, 10, 124-130.

Awh, E., Jonides, J., \& Reuter-Lorenz, P. A. (1998). Rehearsal in spatial working memory. Journal of Experimental Psychology: Human Perception \& Performance, 24, 780-790.

AwH, E., VogeL, E. K., \& OH, S. H. (2006). Interactions between attention and working memory. Neuroscience, 139, 201-208.

BAdDeley, A. D. (1986). Working memory. Cambridge, MA: MIT Press.

BADDEley, A. D., \& Lieberman, K. (1980). Spatial working memory. In R. S. Nickerson (Ed.), Attention and performance VIII (pp. 521-539). Hillsdale, NJ: Erlbaum.

Berger, A., HeniK, A., \& RAFAL, R. [D.] (2005). Competition between endogenous and exogenous orienting of visual attention. Journal of Experimental Psychology: General, 134, 207-221.

BERLUCCHI, G. (2006). Inhibition of return: A phenomenon in search of a mechanism and a better name. Cognitive Neuropsychology, 23, 1065-1074.

Berlucchi, G., Chelazzi, L., \& Tassinari, G. (2000). Volitional covert orienting to a peripheral cue does not suppress cue-induced inhibition of return. Journal of Cognitive Neuroscience, 12, 648-663.

Briand, K. A., Larrison, A. L., \& Sereno, A. B. (2000). Inhibition of return in manual and saccadic response systems. Perception \& Psychophysics, 62, 1512-1524.

Castel, A. D., Pratt, J., \& Craik, F. I. M. (2003). The role of spatial working memory in inhibition of return: Evidence from divided attention tasks. Perception \& Psychophysics, 65, 970-981.

Cheal, M. L., \& Lyon, D. R. (1991). Central and peripheral precuing of forced-choice discrimination. Quarterly Journal of Experimental Psychology, 43A, 859-880.

Deubel, H., \& SchNeider, W. X. (1996). Saccade target selection and object recognition: Evidence for a common attentional mechanism. Vision Research, 36, 1827-1837.

Dorris, M. C., Klein, R. M., Everling, S., \& Munoz, D. P. (2002). Contribution of the primate superior colliculus to inhibition of return. Journal of Cognitive Neuroscience, 14, 1256-1263.

Hoffman, J. E., \& Subramaniam, B. (1995). The role of visual attention in saccadic eye movements. Perception \& Psychophysics, 57, 787-795.

Hunt, A. R., \& Kingstone, A. (2003). Covert and overt voluntary 
attention: Linked or independent? Cognitive Brain Research, 18, 102-105.

JнA, A. P. (2002). Tracking the time-course of attentional involvement in spatial working memory: An event-related potential investigation. Cognitive Brain Research, 15, 61-69.

KLEIN, R. M. (1980). Does oculomotor readiness mediate cognitive control of visual attention? In R. S. Nickerson (Ed.), Attention and performance VIII (pp. 259-276). Hillsdale, NJ: Erlbaum.

KLeIN, R. M. (2000). Inhibition of return. Trends in Cognitive Sciences, 4, 138-147.

Klein, R. M., \& MacInnes, W. J. (1999). Inhibition of return is a foraging facilitator in visual search. Psychological Science, 10, 346-352.

Klein, R. M., \& Pontefract, A. (1994). Does oculomotor readiness mediate cognitive control of visual attention? Revisited! In C. Umiltà $\&$ M. Moscovitch (Eds.), Attention and performance XV (pp. 333350). Cambridge, MA: MIT Press, Bradford Books.

Kowler, E., Anderson, E., Dosher, B., \& Blaser, E. (1995). The role of attention in the programming of saccades. Vision Research, 35, 1897-1916.

LaWrence, B. M., Myerson, J., \& Abrams, R. A. (2004). Interference with spatial working memory: An eye movement is more than a shift of attention. Psychonomic Bulletin \& Review, 11, 488-494.

Lawrence, B. M., Myerson, J., OonK, H. M., \& Abrams, R. A. (2001). The effects of eye and limb movements on working memory. Memory, 9, 433-444.

Loftus, G. R., \& Masson, M. E. J. (1994). Using confidence intervals in within-subjects designs. Psychonomic Bulletin \& Review, 1, 476-490.

Lupiáñez, J., Decaix, C., Sieroff, E., Chokron, S., Milliken, B. \& BARTOLOMEO, P. (2004). Independent effects of endogenous and exogenous spatial cuing: Inhibition of return at endogenously attended target locations. Experimental Brain Research, 159, 447-457.

Lupiáñez, J., Klein, R. M., \& Bartolomeo, P. (2006). Inhibition of return: Twenty years after. Cognitive Neuropsychology, 23, 1003-1014.

Mangun, G. R., Hinrichs, H., Scholz, M., Mueller-Gaertner, H. W., Herzog, H., Krause, B. J., ET AL. (2001). Integrating electrophysiology and neuroimaging of spatial selective attention to simple isolated visual stimuli. Vision Research, 41, 1423-1435.

PosNer, M. I. (1980). Orienting of attention. Quarterly Journal of Experimental Psychology, 32, 3-25.

Posner, M. I., Rafal, R. D., Choate, L. S., \& Vaughan, J. (1985). Inhibition of return: Neural basis and function. Cognitive Neuropsychology, 2, 211-228.
Postle, B. R., Awh, E., Jonides, J., Smith, E. E., \& D’Esposito, M. (2004). The where and how of attention-based rehearsal in spatial working memory. Cognitive Brain Research, 20, 194-205.

Rafal, R. D., Calabresi, P. A., Brennan, C. W., \& Sciolto, T. K. (1989). Saccade preparation inhibits reorienting to recently attended locations. Journal of Experimental Psychology: Human Perception \& Performance, 15, 673-685.

Rizzolatti, G., Riggio, L., Dascola, I., \& Umiltà, C. (1987). Reorienting attention across the horizontal and vertical meridians: Evidence in favor of a premotor theory of attention. Neuropsychologia, 25, 31-40.

Ro, T., FARNe, A., \& CHANG, E. (2003). Inhibition of return and the human frontal eye fields. Experimental Brain Research, 150, 290-296.

SAPIR, A., Soroker, N., Berger, A., \& HeNIK, A. (1999). Inhibition of return in spatial attention: Direct evidence for collicular generation. Nature Neuroscience, 2, 1053-1054.

Sheliga, B. M., Riggio, L., \& Rizzolatti, G. (1994). Orienting of attention and eye movements. Experimental Brain Research, 98, 507-522.

Sheliga, B. M., Riggio, L., \& Rizzolatti, G. (1995). Spatial attention and eye movements. Experimental Brain Research, 105, 261-275.

Shepherd, M., Findlay, J. M., \& Hockey, R. J. (1986). The relationship between eye movements and spatial attention. Quarterly Journal of Experimental Psychology, 38, 475-491.

Tassinari, G., Aglioti, S., Chelazzi, L., Marzi, C. A., \& BerlucCHI, G. (1987). Distribution in the visual-field of the costs of voluntarily allocated attention and of the inhibitory aftereffects of covert orienting. Neuropsychologia, 25, 55-71.

Theeuwes, J., Olivers, C. N. L., \& Chizk, C. L. (2005). Remembering a location makes the eyes curve away. Psychological Science, 16, 196-199.

Theeuwes, J., Van der Stigchel, S., \& Olivers, C. N. L. (2006). Spatial working memory and inhibition of return. Psychonomic Bulletin \& Review, 13, 608-613.

Van der Stigchel, S., \& Theeuwes, J. (2007). The relationship between covert and overt attention in endogenous cuing. Perception \& Psychophysics, 69, 719-731.

(Manuscript received March 25, 2008; revision accepted for publication October 28, 2008.) 